\title{
3D Guided Wave Motion Analysis on Laminated Composites
}

\author{
Zhenhua Tian ${ }^{\mathrm{a}}$, Cara Leckey ${ }^{\mathrm{b}}$ and Lingyu $\mathrm{Yu}^{\mathrm{a}}$ \\ ${ }^{a}$ University of South Carolina, Department of Mechanical Engineering, Columbia SC \\ ${ }^{b}$ Nondestructive Evaluation Sciences Branch, NASA Langley Research Center, Hampton VA
}

\begin{abstract}
Ultrasonic guided waves have proved useful for structural health monitoring (SHM) and nondestructive evaluation (NDE) due to their ability to propagate long distances with less energy loss compared to bulk waves and due to their sensitivity to small defects in the structure. Analysis of actively transmitted ultrasonic signals has long been used to detect and assess damage. However, there remain many challenging tasks for guided wave based SHM due to the complexity involved with propagating guided waves, especially in the case of composite materials. The multimodal nature of the ultrasonic guided waves complicates the related damage analysis. This paper presents results from parallel 3D elastodynamic finite integration technique (EFIT) simulations used to acquire 3D wave motion in the subject laminated carbon fiber reinforced polymer composites. The acquired 3D wave motion is then analyzed by frequencywavenumber analysis to study the wave propagation and interaction in the composite laminate. The frequencywavenumber analysis enables the study of individual modes and visualization of mode conversion. Delamination damage has been incorporated into the EFIT model to generate "damaged" data. The potential for damage detection in laminated composites is discussed in the end.
\end{abstract}

Keywords: Frequency wavenumber analysis, EFIT, delamination, guided waves PACS: $43.20 \mathrm{Ks}$

\section{INTRODUCTION}

Current composite "safety margin" design comes with a weight penalty which would be mitigated by the development of accurate and efficient structural health monitoring (SHM) methods to locate and quantify the unique damage types that can occur in composite materials (e.g., microcracking and delaminations). Unexpected damage can occur in aerospace composites due to impact events or due to stressing of the material during off-nominal loading events. The problem of how best to detect such material changes in composite components is still being solved, and a solution to this problem will be imperative for safe and functional optimally-designed next-generation composite aircraft.

Ultrasonic guided waves have proved useful for structural health monitoring (SHM) and nondestructive evaluation (NDE) due to their ability to propagate long distances with less energy loss compared to bulk waves and due to their sensitivity to small defects in the structure [1-5]. Analysis of actively transmitted ultrasonic signals is a conventional NDE methodology that has long been used to detect and assess damage in aerospace components. Many researchers have contributed to the study of SHM using guided wave propagation methods [2-10]. Although recent advances in guided wave based SHM technology have demonstrated the feasibility of detecting and locating damage in composite structural components, such as [11-13], there remain many challenging tasks due to the complexity involved with propagating guided waves.

In this paper we present our studies on guided wave propagation in anisotropic carbon fiber reinforced polymer (CFRP) materials using wavenumber based data analysis methods. In our study, the waveforms are acquired from three-dimensional (3D) elastodynamic finite integration technique (EFIT) that generates both in-plane and out-ofplane velocity components. Utilizing wavenumber processing methods, wave interaction with delamination damage is studied. The two-dimensional Fourier transform (2D FT) is applied to time-space wavefield data such that they are converted to the frequency-wavenumber domain where the guided wave propagation characteristics can be assessed more intuitively. A short space 2D FT is also developed and applied to generate a space-wavenumber relationship. The wave interaction and mode conversion at the delamination region are revealed using these wavenumber processing methods.

\section{FREQUENCY-WAVENUMBER ANALYSIS}

The Fourier transform has proved to be an enormously useful tool for analyzing time series. The concept of Fourier analysis is straightforwardly extended to multidimensional signals such as the time-space wavefield, which 
is in terms of the time variable $t$ and the space variable $x$. Frequency-wavenumber representation can be obtained by applying the 2D FT to the time-space wavefield $u(t, x)$, as [14-16]

$$
U(f, k)=\mathrm{F}_{2 \mathrm{D}}[u(t, x)]=\int_{-\infty}^{\infty} \int_{-\infty}^{\infty} u(t, x) e^{-j(2 \pi f t-k)} d t d x
$$

where $U(f, k)$ is the frequency-wavenumber spectrum which can be interpreted as an alternative representation of the time-space wavefield $u(t, x)$ in terms of the frequency variable $f$ and the wavenumber $k$.

Figure 1 gives an example of frequency-wavenumber analysis of a simulated harmonic time-space wavefield $u_{0}(t$, $x$ ) with a constant unit amplitude (Figure 1(a)). Figure 1(b) gives the frequency-wavenumber spectrum in terms of amplitude (color map) versus frequency and wavenumber. The spectrum has a maximum value at $(150 \mathrm{kHz}, 0.68$ $\mathrm{rad} / \mathrm{mm}$ ), indicating the harmonic (frequency, wavenumber) component. Note that the small ripples around the spectrum center $(150 \mathrm{kHz}, 0.68 \mathrm{rad} / \mathrm{mm})$ are caused by Fourier transform leakage.
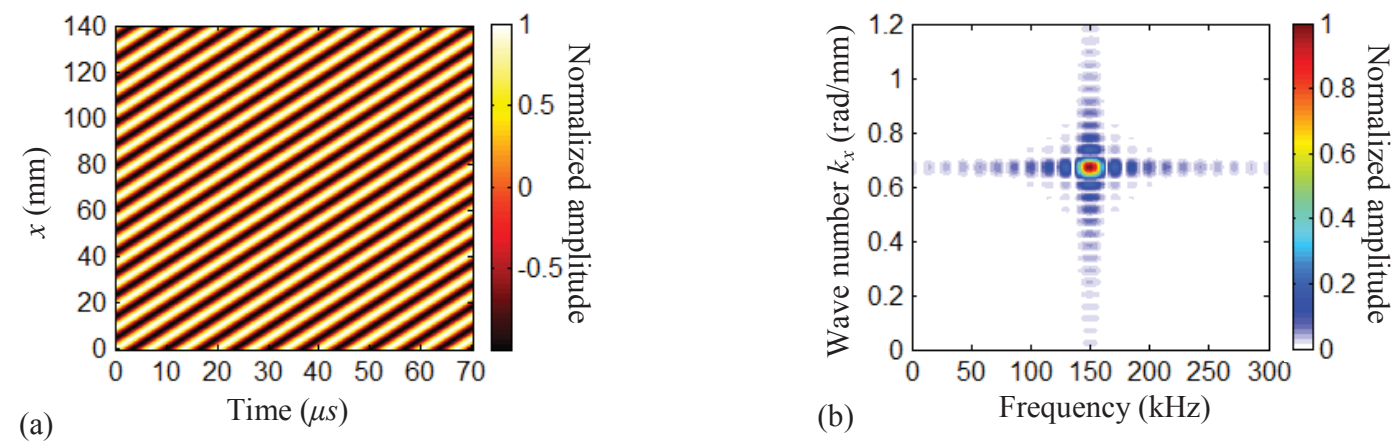

FIGURE 1 2D FT of a single frequency single mode numerical wavefield $u(t, x)$ : (a) time-space wavefield; (b) frequency-wavenumber spectrum.

\section{SPACE-FREQUENCY-WAVENUMBER ANALYSIS}

The frequency-wavenumber analysis unveils wave propagation characteristics that cannot be explicitly seen in the time-space domain. However, this approach does not retain the spatial information. Through a spatial windowing technique, spatial information can be retained and the relationship between location and frequency/wavenumber can be revealed.

Similar to the idea of the short time Fourier transform which uses a windowing technique [17], a short space 2D FT is developed to perform the space-frequency-wavenumber analysis which can retain the spatial information. By this means, frequency-wavenumber spectra at various spatial locations are obtained, resulting in a three-dimensional space-frequency-wavenumber spectrum of the wavefield. The short space 2D FT of a wavefield $u(t, x)$ is expressed as:

$$
S(\alpha, f, k)=\int_{-\infty}^{\infty} \int_{-\infty}^{\infty} u(t, x) W^{*}(t, x-\alpha) e^{-j(2 \pi f t-k x)} d t d x
$$

where $\alpha$ is the space index and $W(t, x)$ is the $2 \mathrm{D}$ window function. In our study, a Hanning function is used to construct the $2 \mathrm{D}$ window $W(t, x)$, giving:

$$
W(t, x)=\left\{\begin{array}{cc}
0.5\left[1+\cos \left(2 \pi \frac{x}{D_{x}}\right)\right] & \text { if }|x| \leq D_{x} / 2 \\
0 & \text { otherwise }
\end{array}\right.
$$

where $D_{x}$ is the window length in the space dimension. The 2D FT of the windowed portion will yield a frequencywavenumber spectrum, which only contains the local information of the windowed region. By sliding the window along the space dimension, the resulting space-frequency-wavenumber spectrum can indicate how the frequencywavenumber components vary in space.

Figure 2 shows an example of the short space 2D FT for the single frequency single mode time-space wavefield $u_{0}(t, x)$ in Figure 1(a). An illustration of the moving window is shown in Figure 2(a). By sliding the window along the space dimension and stacking the corresponding frequency-wavenumber spectra, a space-frequency- 
wavenumber spectrum is obtained. Figure 2(b) plots the resulting space-wavenumber spectrum at a selected frequency of $150 \mathrm{kHz}$. The spectrum shows wavenumber as a function of propagation distance.

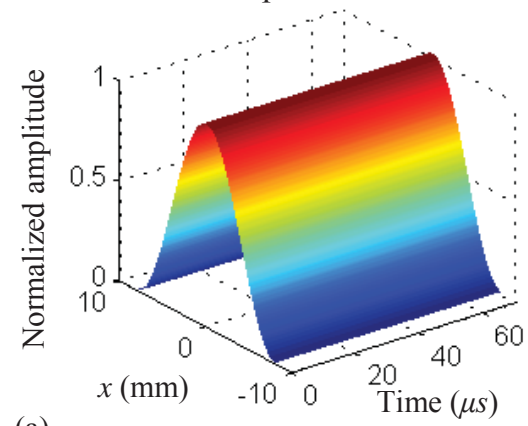

(a)

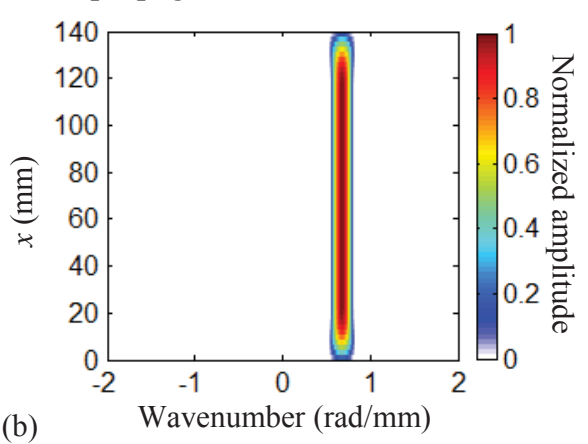

(b)

FIGURE 2 Short space 2D FT of a single frequency single mode numerical wavefield $u_{0}(t, x)$ : (a) time-space window; (b) space-wavenumber spectrum at the frequency $150 \mathrm{kHz}$.

\section{EFIT WAVE MOTION SIMULATION}

3D EFIT simulations provided simulated guided wave data for the studies presented in this paper. Previous work by the authors has demonstrated how 3D simulated guided wave data can aid in the study of guided wave interaction with damage in isotropic and anisotropic materials[16, 18, 19]. Since the anisotropic EFIT equations are quite lengthy they are not repeated here, but can be found in prior work by Halkjaer [20]. The EFIT code implemented for this paper is custom parallelized code that runs efficiently on cluster computing resources. Additional details about the anisotropic EFIT simulation code, including results of code validation studies can be found in prior work by one of the authors [19].

\section{Wave simulation setup}

Wave propagation in an 8-ply IM7/8552 composite laminate with layup $\left[0_{2} / 90_{2}\right]_{\mathrm{s}}$ was simulated for two cases: 1 ) an undamaged composite laminate, 2) a composite laminate with a delamination (void) damage located between uppermost $0 / 90$ plies (closest to the plate surface where the incident wave excitation is applied). The specimen layout is given in Figure 3. The composite layup was chosen to match composite samples that were fabricated at NASA LaRC and will be used in future work. In order to isolate mode conversion behavior from more complex scattering and geometric effects, long rectangular delaminations of varying lengths were inserted into the simulation for the delamination studies. In future work the authors plan to insert square delaminations to match the shape, size, and location of Teflon inserts in a NASA LaRC composite sample. This approach allows for the step-by-step establishment of a clear understanding of guided wave behavior and wavenumber analysis techniques for composite materials containing delaminations. In future work it will be important to move on to study more realistic damage geometries.

The EFIT code incorporates ply-level material properties, and the lay up is simulated by inputting the appropriately rotated ply level material properties for each layer. The material properties (single ply layer) used in the EFIT simulations are shown in Table 1, where $\mathrm{E}_{1}$ is in the fiber direction. In order to allow for two spatial steps per ply, the spatial step size of the EFIT simulation was set smaller than the minimal requirement of $\Delta \mathrm{x} \leq \lambda_{\min } / 10$ to $\Delta \mathrm{x}=53.3 \mu \mathrm{m}\left(\sim \lambda_{\min } / 69\right.$ based on dispersion curves for the pristine laminate). For the damaged composite specimen the $53.3 \mu \mathrm{m}$ step size is equivalent to $\sim \lambda_{\min } / 31$ in the thinned region above the delamination. The wavelength to step size ratio above the delamination is expected to be adequate for accurately calculating wave behavior based on the results of prior validation comparisons where a similar ratio was used [19]. The total thickness of the simulated laminate is $0.854 \mathrm{~mm}$. Additionally, we note that the temporal step size (4.1 ns) meets stability requirements [19]. The delamination was incorporated by implementing stress free boundary conditions at the damage location. The simulation incident wave was a $300 \mathrm{kHz} 3$-cycle Hanning windowed sine wave. The excitation was introduced to the simulation space as a $7 \mathrm{~mm}$ diameter ring-type excitation on the top laminate surface in order to approximate the behavior of a $7 \mathrm{~mm}$ piezoelectric wafer [18]. 
TABLE 1. Material properties of the lamina [21]

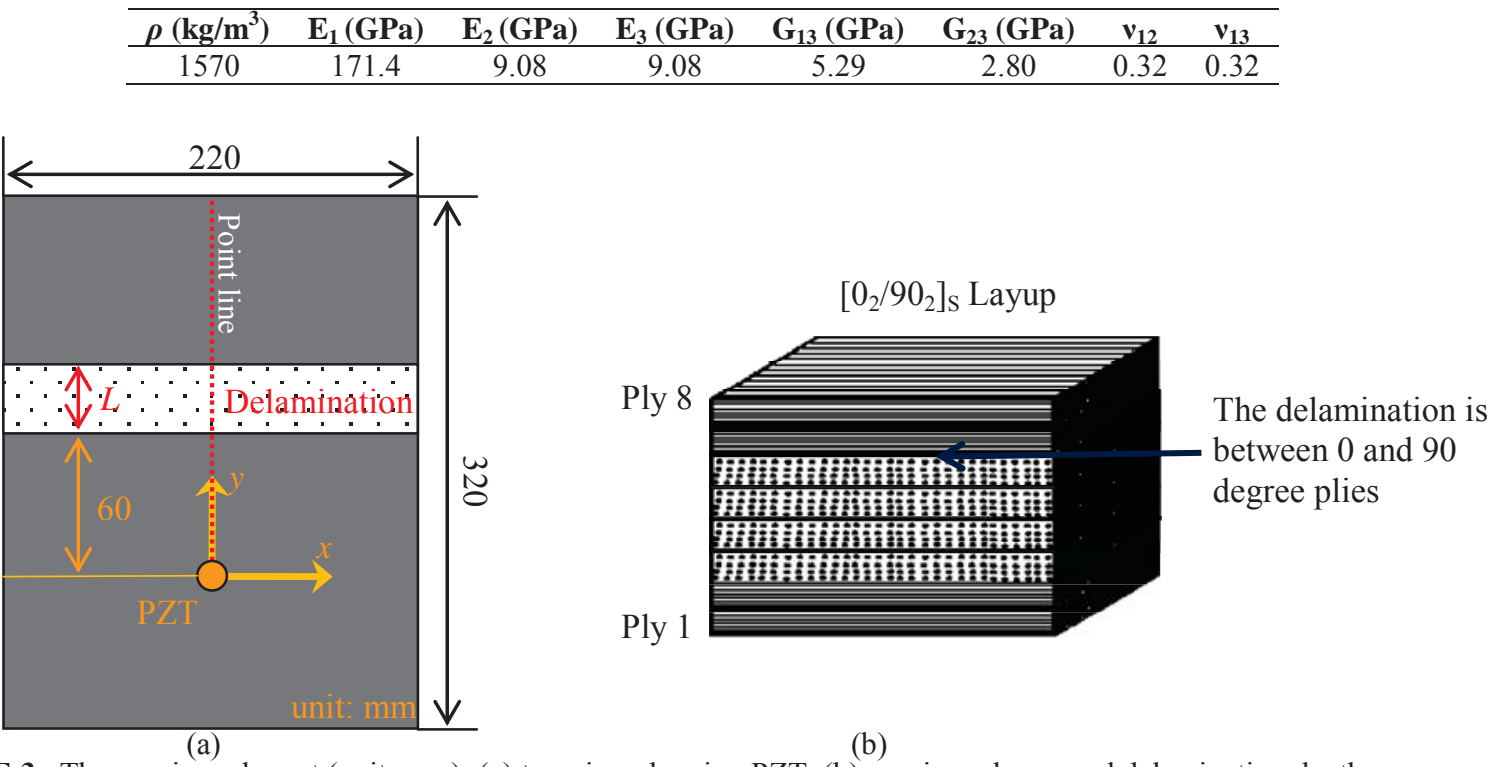

FIGURE 3 The specimen layout (unit: $\mathrm{mm}$ ): (a) top view showing PZT, (b) specimen layup and delamination depth.
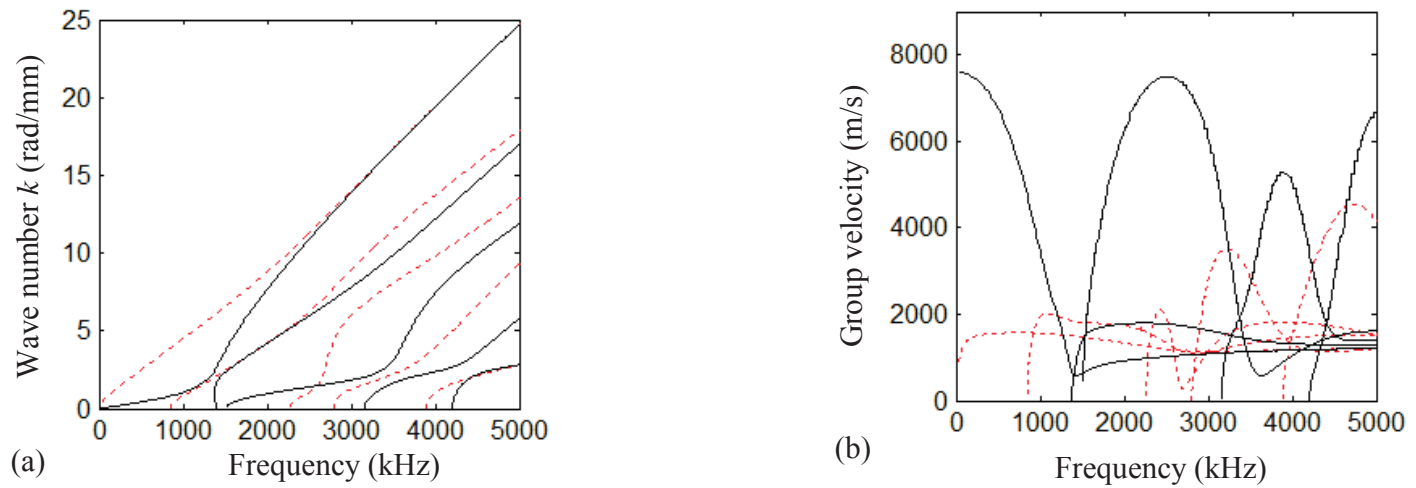

FIGURE 4 Theoretical dispersion curves from DISPERSE ${ }^{\mathrm{TM}}$ : (a) frequency-wavenumber dispersion curves (90 deg); (b) group velocity dispersion curves (90 deg).

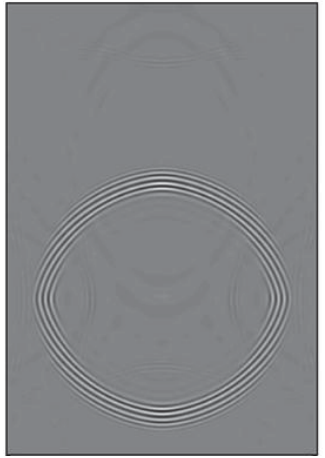

(a)

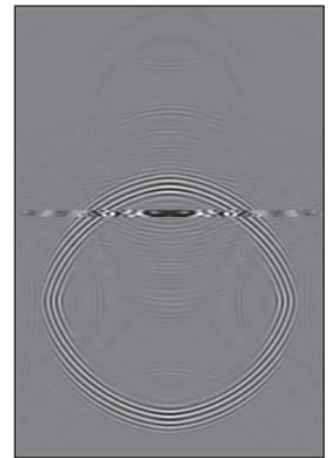

(b)

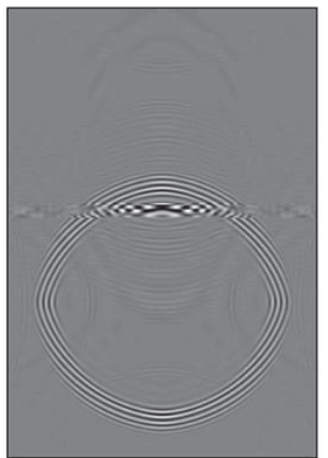

(c)

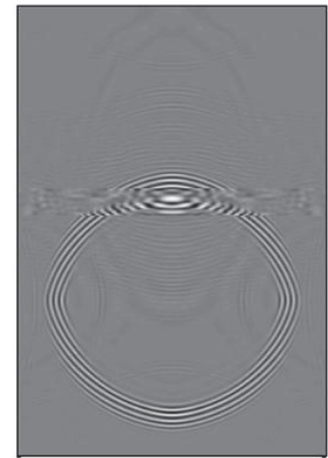

(d)

FIGURE 5 EFIT simulation out-of-plane (OOP) results at time $t=24.3 \mu$ s on the for the top surface of the composite plate: (a) pristine plate; (b) with $5 \mathrm{~mm}$ delamination; (c) with $10 \mathrm{~mm}$ delamination; (d) with $20 \mathrm{~mm}$ delamination. 


\section{Simulation results}

Full wavefield velocity data (in-plane $v_{x}$ and $v_{y}$ components and out-of-plane $v_{z}$ component) were output from the EFIT simulation for the entire top surface of the simulated composite sample. The surface velocity data was output every 20 time steps in order to reduce the size of the resulting data set. This time output meets sampling requirements of the data analysis techniques.

Dispersion curves for the $\left[0_{2} / 90_{2}\right]_{\mathrm{s}}$ were calculated using DISPERSE software*, and results for the 90 deg fiber direction (corresponding to the point-line in Figure 3 and the results in the following section) are shown in Figure 4. At the selected frequency-thickness of the simulation, two guided wave modes are expected to exist.

Figure 5 shows time domain wavefield images at a single point in time from EFIT simulations of the pristine sample and for delamination damage of increasing length, as indicated in the figure caption. The $\mathrm{A}_{0}$ mode is clearly observed from the out-of-plane wavefield images. The faster $\mathrm{S}_{0}$ mode has much lower out-of-plane amplitude and is not readily visible in the images. Wave interactions with the delamination regions can be observed in the three damage scenarios, however, specific behaviors such as mode conversion and wavenumber shifts, are not clear from the time domain wavefield images.

\section{WAVE INTERACTION WITH DELAMINATION}

In this section, the 3D in-plane and out-of-plane wave motion data generated by EFIT model are analyzed with the frequency-wavenumber and space-frequency-wavenumber approaches in order to understand the wave propagation characteristics and wave interaction with the delamination damage. The wave propagation along the $90^{\circ}$ direction (the point line in Error! Reference source not found.), which passes through the delamination area, is studied. Since the $v_{x}$ component is nearly zero, here we show only the in-plane $v_{y}$ and out-of-plane $v_{z}$ components.
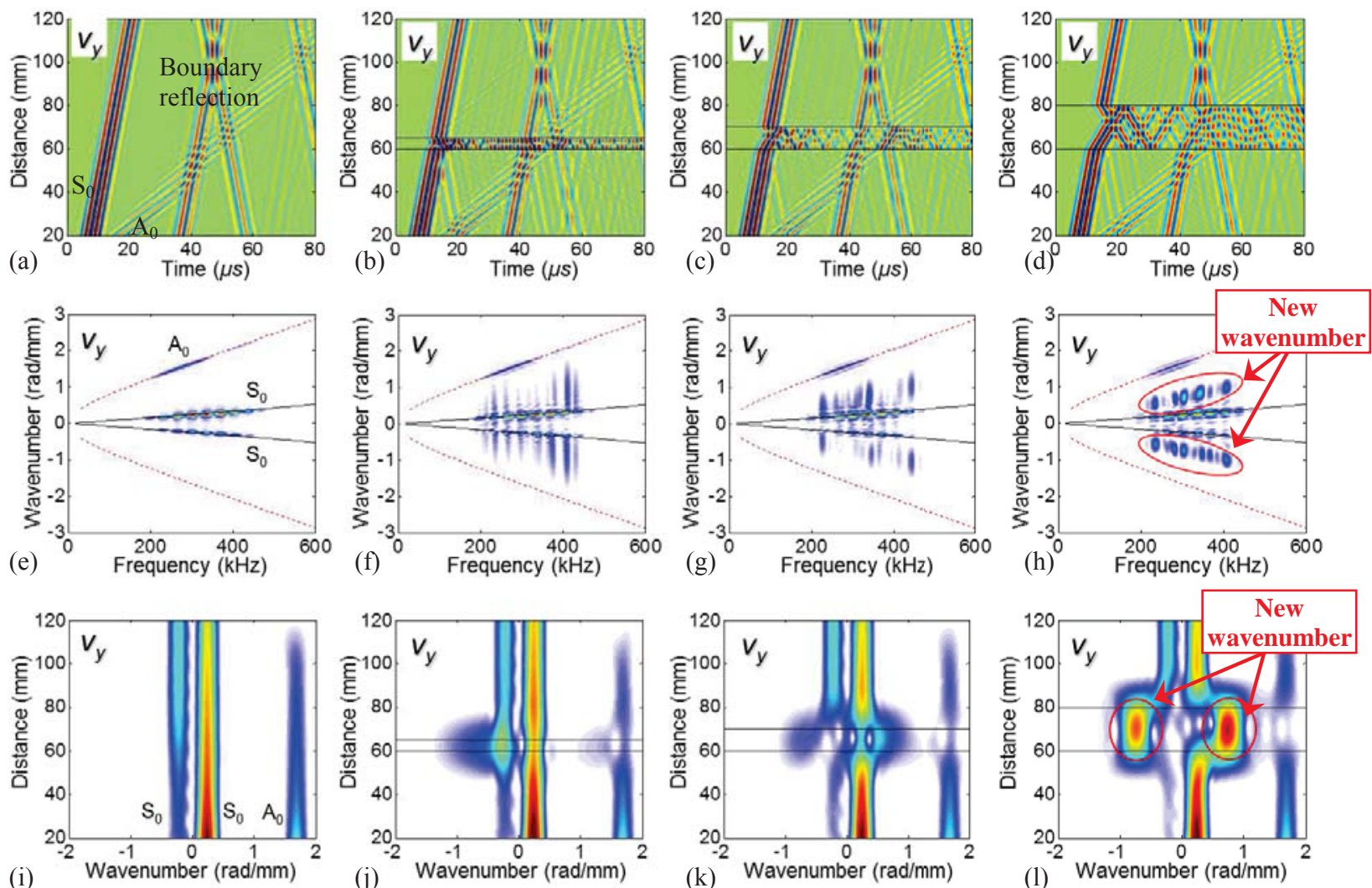

FIGURE 6 In-plane $v_{y}$ results: (a), (b), (c) and (d) are the time-space wavefields for the pristine plate, plate with $L=5 \mathrm{~mm}$ delamination, plate with $L=10 \mathrm{~mm}$ delamination and plate with $L=20 \mathrm{~mm}$ delamination; (e), (f), (g) and (h) are the frequency wavenumber spectra corresponding to the time-space wavefields in (a), (b), (c) and (d), respectively; (i), (j), (k) and (l) are the space-wavenumber spectra corresponding to the wavefields in (a), (b), (c) and (d), respectively.

\footnotetext{
${ }^{*}$ http://www3.imperial.ac.uk/nde/products\%20and\%20services/disperse
} 


\section{In-plane $v_{y}$ component}

The time-space wavefields of the in-plane $v_{y}$ component for pristine and delamination cases containing different delamination sizes $(L=5,10$ and $20 \mathrm{~mm})$ are shown in Figure 6(a)-(d). The wavefield is plotted as amplitude (in color) versus time $t$ and propagating distance $x$ with respect to the actuator position. A fast $\mathrm{S}_{0}$ mode and a slow $\mathrm{A}_{0}$ mode are observed in the pristine plate. The in-plane amplitude of $\mathrm{S}_{0}$ mode is higher than that of $\mathrm{A}_{0}$ mode, as expected. For the delamination cases, it can be observed that the waves propagate forward and backward within the delamination region and the energy is somewhat "trapped" but slowly "leaking" from the damage region. This observation is consistent with what has been reported previously in the literature [13]. Although not readily apparent with the color scale used in figure 6 , we also note that as the $\mathrm{S}_{0}$ mode impinges and reflects from the delamination edge, an $\mathrm{A}_{0}$ mode is created due to mode conversion.

In order to leverage frequency-wavenumber information, both 2D FT and short-space 2D FT analyses are applied to the data. A window size of $50 \mathrm{~mm}$ (twice the wavelength of the $\mathrm{S}_{0}$ mode in the full plate thickness) was used for the short-space 2D FT, in order to obtain good space-wavenumber resolution. Using the 2D FT, the time space wavefield is transformed into the frequency-wavenumber domain. The frequency-wavenumber spectra of the wavefields for each case are given in Figure 6(e)-(h). The solid black line and red dashed line are theoretical dispersion curves. In the spectrum of the pristine plate, both $\mathrm{A}_{0}$ and $\mathrm{S}_{0}$ mode can be seen, with $\mathrm{S}_{0}$ being stronger for the in-plane data. The predicted modes are consistent with the time-space wavefield observations. In the delamination cases additional wavenumber components (both positive and negative components, representing forward and backward wave propagation) appear. In Figure 6(h), we can clearly observe a new frequencywavenumber component which may be related to the waves trapped inside the delamination region.
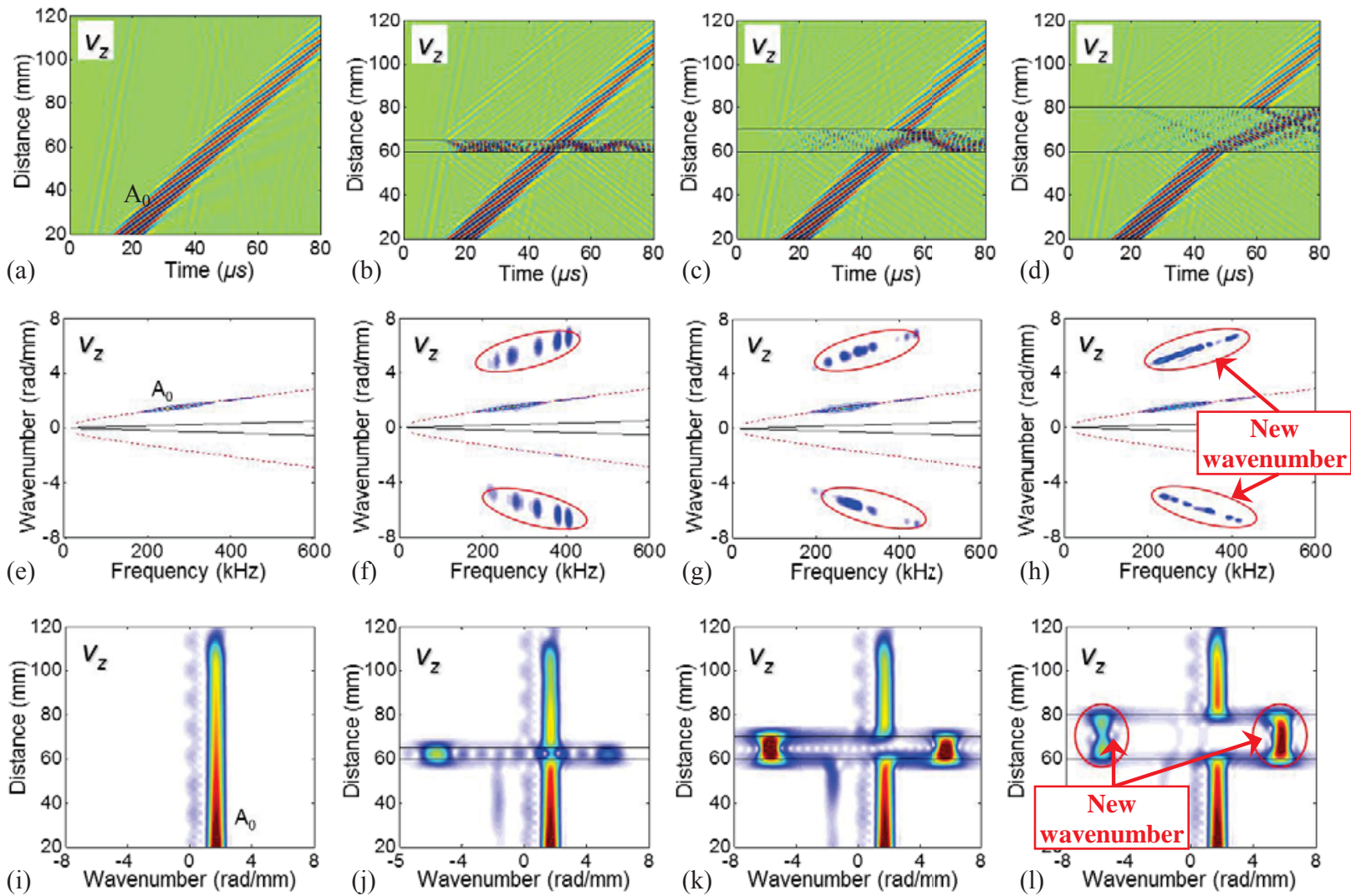

FIGURE 7 Out-of-plane $v_{z}$ results: (a), (b), (c) and (d) are the time-space wavefields for the pristine plate, plate with $L=5 \mathrm{~mm}$ delamination, plate with $L=10 \mathrm{~mm}$ delamination and plate with $L=20 \mathrm{~mm}$ delamination; (e), (f), (g) and (h) are the frequency wavenumber spectra corresponding to the time-space wavefields in (a), (b), (c) and (d), respectively; (i), (j), (k) and (l) are the space-wavenumber spectra corresponding to the wavefields in (a), (b), (c) and (d), respectively. 
Using short-space 2D FT, the space information can be retained, in addition to the wavenumber information. Figures 6(i)-(1) present space-wavenumber spectra at $300 \mathrm{kHz}$. As shown in Figure 6(1), within the delamination region, there exist new wavenumber components (again including both positive and negative wavenumbers representing forward and backward wave propagation). The center of the new wavenumber component is around $0.75 \mathrm{rad} / \mathrm{mm}$ which is close to the theoretical wavenumber $(0.78 \mathrm{rad} / \mathrm{mm})$ for the $\mathrm{S}_{0}$ mode in the upmost two ply layers (i.e., above the delamination).

\section{Out-of-plane $v_{\mathrm{z}}$ component}

The time-space wavefields of out-of-plane $v_{z}$ component for the pristine and delamination cases (with delamination sizes $L=5,10$ and $20 \mathrm{~mm}$ ) are shown in Figure 7(a)-(d). A strong $\mathrm{A}_{0}$ mode can be clearly seen in the wavefield of pristine plate in

Figure 7(a). In contrast, the out-of-plane amplitude of $\mathrm{S}_{0}$ mode is very weak. At the delamination region, wave interactions with the delamination appear.

The frequency-wavenumber spectra are shown in Figure 7(e)-(h). From the spectrum of the pristine plate, a clear $\mathrm{A}_{0}$ mode can be seen. As in the $\mathrm{v}_{\mathrm{y}}$ results, additional wavenumber components (both positive and negative components representing forward and backward wave propagation) appear in the delamination cases. Figure 7(i)-(l) shows space-wavenumber spectra at $300 \mathrm{kHz}$ obtained by short-space 2D FT. A window size of $8 \mathrm{~mm}$, twice the wavelength of the $A_{0}$ mode in the full plate thickness, was used for the short-space 2D FT. As shown in Figure 7(1), the new wavenumber components only exist within the delamination region, which means this new wavenumber components are related to the waves propagating within the delamination region. The center of the new wavenumber component is around $5.6 \mathrm{rad} / \mathrm{mm}$. However, this wavenumber does not match closely with the theoretical wavenumber $(3.8 \mathrm{rad} / \mathrm{mm})$ of the $\mathrm{A}_{0}$ mode in the upmost two ply layers. The reason for this apparent mismatch is unclear. The $\mathrm{A}_{0}$ mode is highly dispersive in the corresponding frequency-thickness range, and the bandwidth of the incident excitation could contribute to the mismatch. Another potential reason could be that the simulation spatial step size is not adequate for capturing the short wavelength behavior above the delamination (though recall that the step size is equal to $\sim \lambda_{\min } / 31$ above the delamination). This unexpected mismatch will be further investigated in future work.

\section{CONCLUSIONS}

This paper presents our studies on guided wave propagation in CFRP plates using wavenumber-based data analysis methods. The frequency-wavenumber analysis enables determination of all existing wave modes while the space-wavenumber analysis reveals how the wavenumber component varies with respect to the propagation distance. 3D EFIT was used to simulate guided wave propagation in pristine and delaminated CFRP composites, generating time-space wavefields of the in-plane and out-of-plane velocity components. Waves are seen propagating within the delamination area at different velocities from the pristine case and in both forward and backward directions.

Using the frequency-wavenumber and space-frequency-wavenumber analysis techniques, the wave interactions with delamination damage are more easily analyzed than when simply relying on time-space wavefields. Frequencywavenumber results from both in-plane and out-of-plane motion studies show that there are new wavenumber components in the delaminated plate compared to those of the pristine plate. The space-wavenumber spectrum then reveals that the new wavenumber components appear within the delamination area. This finding is consistent with the standing waves observed in the wavefield images reported in the literature [13]. From the in-plane motion study, the new wavenumber component is consistent with the expected $\mathrm{S}_{0}$ wavenumber for the upmost two ply layers. However, from the out-of-plane study, the new wavenumber component shows an apparent mismatch from the expected $\mathrm{A}_{0}$ mode wavenumber in the upmost two ply layers. Further studies will be conducted to explore these results.

\section{ACKNOWLEDGMENTS}

Part of this work is conducted through the non-reimbursement Space Act Agreement SAA1-1181 between South Carolina Research Foundation (SCRF) and the National Aeronautics and Space Administration (NASA) Langley Research Center. This work was partially supported by SPARC Graduate Fellowship from the Office of the Vice President for Research at the University of South Carolina 


\section{REFERENCES}

1. J. L. Rose, Ultrasonic Waves in Solid Media, Cambridge University Press, 1999.

2. D. C. Worlton, Nondestructive Testing, 15, 218-222 (1957).

3. D. N. Alleyne and P. Cawley, IEE Transactions on Ultrasonics Ferroelectrics and Frequency Control, 39, 381-397 (1992).

4. R. P. Dalton, P. Cawley and M. J. S. Lowe, Journal of Nondestructive Evaluation, 20, 29-46 (2001).

5. W. H. Ong and W. K. Chiu, Smart Materials Research, 2012, 1-9 (2012).

6. D. N. Alleyne and P. Cawley, Ndt \& E International, 25, 11-22 (1992).

7. P. S. Tua, S. T. Quek and Q. Wang, Smart Materials \& Structures, 13, 643-660 (2004).

8. J. Rajagopalan, K. Balasubramaniam and C. V. Krishnamurthy, Smart Materials \& Structures, 15, 1190-1196 (2006).

9. S. R. Anton, G. Park, C. R. Farrar and D. J. Inman, Proceedings of SPIE 2007, Health Monitoring of Structural and Biological System, 6532, 65320B (2007).

10. W. J. Staszewski, B. C. Lee and R. Traynor, Measurement Science \& Technology, 18, 727-739 (2007).

11. C. T. Ng and M. Veidt, Smart Materials \& Structures, 18, 074006 (2009).

12. A. S. Purekar and D. J. Pines, Journal of Intelligent Material Systems and Structures, 21, 995-1010 (2010).

13. H. Sohn, D. Dutta, J. Y. Yang, H. J. Park, M. P. DeSimio, S. E. Olson and E. D. Swenson, Composites Science and Technology, 71, 1250-1256 (2011).

14. M. Ruzzene, Smart Materials and Structures, 16, $2116-2129$ (2007)

15. T. E. Michaels, J. E. Michaels and M. Ruzzene, Ultrasonics, 51, 452-466 (2011).

16. L. Yu, C. Leckey and Z. Tian, Smart Materials \& Structures, 22, 1-12 (2013).

17. L. Cohen, Time Frequency Analysis: Theory and Applications, Prentice-Hall Inc., 1994.

18. L. Yu and C. Leckey, Journal of Intelligent Material Systems and Structures, (2013).

19. C. Leckey, M. D. Rogge and F. R. Parker, Ultrasonics, in press, DOI: 10.1016/j.ultras.2013.05.007 (2013).

20. S. Halkjaer, "Elastic wave propagation in anisotropic inhomogeneous materials", Ph.D. Thesis, Technical University of Denmark, 2000.

21. P. P. Camanho, P. Maimi and C. G. Davila, Composite Science and Technology, 67, 2715-2727 (2007). 